\title{
Dried Leaf Artemisia Annua Improves Bioavailability of Artemisinin via Cytochrome P450 Inhibition and Enhances Artemisinin Efficacy Downstream
}

\author{
Matthew R. Desrosiers ${ }^{1}$ (D) , Alexis Mittleman ${ }^{2}$ and Pamela J. Weathers ${ }^{1, *}$ \\ 1 Department of Biology and Biotechnology, Worcester Polytechnic Institute, 100 Institute Road, Worcester, \\ MA 01609, USA; mrdesrosiers@wpi.edu \\ 2 Department of Biomedical Engineering, Worcester Polytechnic Institute, 100 Institute Road, Worcester, \\ MA 01609, USA; amittelman@wpi.edu \\ * Correspondence: weathers@wpi.edu; Tel.: +1-508-831-5196
}

Received: 27 December 2019; Accepted: 5 February 2020; Published: 7 February 2020

check for updates

\begin{abstract}
Artemisia annua L. and artemisinin, have been used for millennia to treat malaria. We used human liver microsomes (HLM) and rats to compare hepatic metabolism, tissue distribution, and inflammation attenuation by dried leaves of $A$. annua (DLA) and pure artemisinin. For HLM assays, extracts, teas, and phytochemicals from DLA were tested and $\mathrm{IC}_{50}$ values for CYP2B6 and CYP3A4 were measured. For tissue distribution studies, artemisinin or DLA was orally delivered to rats, tissues harvested at $1 \mathrm{~h}$, and blood, urine and feces over $8 \mathrm{~h}$; all were analyzed for artemisinin and deoxyartemisinin by GC-MS. For inflammation, rats received an intraperitoneal injection of water or lipopolysaccharide (LPS) and $70 \mathrm{mg} / \mathrm{kg}$ oral artemisinin as pure drug or DLA. Serum was collected over $8 \mathrm{~h}$ and analyzed by ELISA for TNF- $\alpha$, IL-6, and IL-10. DLA-delivered artemisinin distributed to tissues in higher concentrations in vivo, but elimination remained mostly unchanged. This seemed to be due to inhibition of first-pass metabolism by DLA phytochemicals, as demonstrated by HLM assays of DLA extracts, teas and phytochemicals. DLA was more effective than artemisinin in males at attenuating proinflammatory cytokine production; the data were less conclusive in females. These results suggest that the oral consumption of artemisinin as DLA enhances the bioavailability and anti-inflammatory potency of artemisinin.
\end{abstract}

Keywords: Artemisia annua; artemisinin; cytochrome P450 inhibition; tissue distribution; inflammation

\section{Introduction}

In 2017, there were 219 million cases and 435,000 deaths from malaria according to the World Health Organization [1]. The frontline treatment is artemisinin combination therapy (ACT) that relies on semisynthetic derivatives of the drug artemisinin plus a codrug to eliminate patient parasites [2]. While effective, ACTs are often not financially feasible to those most in need in Africa, where $93 \%$ of malaria deaths occur [1]. Thus, there is great need for a more cost-effective alternative to ACTs that can treat malaria among the poorest of the world.

The Chinese herb, Artemisia annua L., naturally produces and stores artemisinin (Figure 1A) in the glandular trichomes on its leaves, stems, and flowers [3]. Used for centuries as a tea infusion to treat a variety of illnesses including malaria [4], recent clinical data suggested that carefully prepared tea infusions made from the plant were as effective or better at eliminating malaria parasites as ACTs $[5,6]$. Furthermore, artemisinin delivered as a tea infusion or as dried leaves of A. annua (DLA) is significantly more bioavailable, crosses the intestine more efficiently, and has about four-fold greater solubility than pure artemisinin [7-9]. DLA also does not require extraction and purification, making it an affordable alternative to ACTs. 


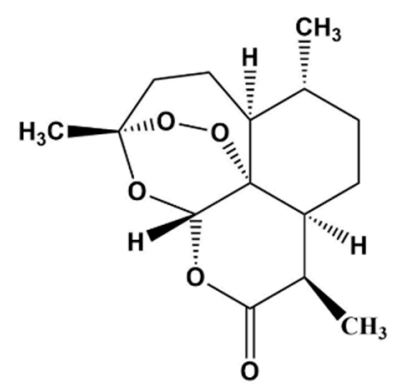

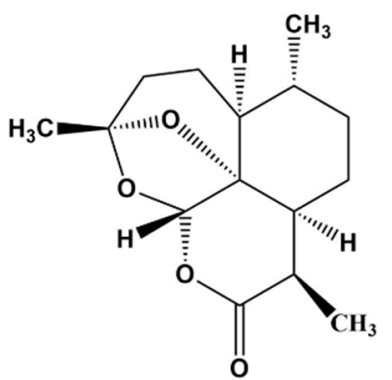

Figure 1. Chemical structures of artemisinin (A) and deoxyartemisinin (B).

Artemisinin is metabolized by liver cytochrome P450s (CYPs), mainly CYP2B6 with a minor contribution from CYP3A4, to yield deoxyartemisinin (Figure 1B), crystal 7, deoxydihydroartemisinin, and 9,10 dihydrodeoxyartemisinin [10,11], all of which are therapeutically inactive. While the enhanced bioavailability of artemisinin afforded by DLA is partially attributed to increased solubility and intestinal transport $[7,8]$, we posit that inhibition of CYP2B6 and/or CYP3A4 by DLA phytochemicals also plays a role in significantly increasing artemisinin bioavailability because more artemisinin would pass through the liver unmetabolized. In turn, that should also increase artemisinin distribution in tissues and organs with greater impact on biological responses, e.g., effects on inflammation.

Despite significant bioavailability and pharmacokinetic differences between pure artemisinin and DLA-delivered artemisinin $[9,12,13]$, to our knowledge, no studies have been done to determine absorption, distribution, metabolism and excretion (ADME) of the drug in vivo when delivered as DLA. There is only one report that determined tissue distribution of orally delivered pure artemisinin; it used a semiquantitative thin-layer chromatography (TLC) densitometric method to quantify artemisinin [14]. We are also not aware of any studies to determine in vivo gender differences in ADME of DLA-delivered artemisinin [15]. One study did show that the area under the curve (AUC) for pure artemisinin was two-fold higher in female rats compared to males after intraperitoneal administration. That same study showed artemisinin disappearance was 3.9-fold higher in liver microsomes from male vs. female livers, suggesting that artemisinin metabolism is gender dependent [15].

Artemisinin and other A. annua phytochemicals also have anti-inflammatory properties and are being investigated as therapeutics for several inflammatory diseases [16-19]. Artemisinin attenuates inflammation by blocking NF- $\mathrm{kB}$ and MAPK signaling pathways that lead to inflammatory cytokine production [16]. A. annua also produces other anti-inflammatory phytochemicals, including flavonoids and monoterpenes. The flavonoids, casticin and chrysosplenol-D, reduced in vitro and in vivo inflammation [20], and the monoterpene, 1,8-cineol (eucalyptol) inhibited the production of six inflammatory cytokines in vitro [21]. A. аnnua also produces rosmarinic and chlorogenic acids, each of which has anti-inflammatory activity [22]. With this rich mixture of anti-inflammatory phytochemicals, it was expected that $A$. annua could provide an alternative cost-effective therapeutic for inflammatory diseases. However, few in vivo studies have investigated whole plant $A$. annua treatment for inflammation.

The present study shows that artemisinin consumed orally as A. annua plant material (DLA) is more bioavailable from inhibition of the important artemisinin-metabolizing enzymes, CYP2B6 and CYP3A4, by compounds produced in the plant. Downstream of this first pass metabolism, DLA-delivered artemisinin is greater in tissues and organs and inflammation is decreased compared to pure artemisinin. We also show significant differences in gender with female rats absorbing higher amounts of artemisinin in several tissues regardless of delivery as DLA or pure drug. Together, these results better explain how per os DLA-delivered artemisinin bioavailability is greater than that from per os pure artemisinin. 


\section{Materials and Methods}

\subsection{Plant Material}

We used dried leaves of the SAM cultivar of Artemisia annua L. (voucher MASS 317314) and Artemisia afra (SEN) (voucher LG0019529). SAM and SEN contained artemisinin between 1.0-1.2\% and $0.02 \%$, and flavonoid contents of $0.93-0.63 \%$ and $0.71 \%(w / w)$, respectively. A. annua was grown and processed as detailed in Weathers et al. 2014 [23]. A. afra was grown in Senegal and dried leaves were provided as a gift from Guy Mergaei, Université de Liège, Belgium. Artemisinin content was determined via GC-MS as detailed in Weathers and Towler 2012 [24]. Total flavonoid content was measured by the $\mathrm{AlCl}_{3}$ method of Arvouet-Grand et al. 1994 [25]. The A. annua used remains consistent in its phytochemical content over time as detailed in Weathers and Towler 2014 [26] and Gruessner et al. 2019 [27]. The A. afra used was from one batch of dried leaves for all experiments and was thus phytochemically consistent. Extensive phytochemical profiles of the A. annua and A. afra dried leaf materials used in this study are detailed in Weathers and Towler 2014 [26] and Munyangi et al. 2018 [28], respectively.

\subsection{Chemicals and Reagents}

Chemicals and reagents were from Sigma Aldrich (St. Louis, MO, USA) unless otherwise stated. Artemisinin was from Cayman Chemical (Ann Arbor, MI, USA); deoxyartemisinin from Toronto Research Chemicals (North York, ON, Canada). Lipopolysaccharide was Escherichia coli serotype O111:B4 and dissolved in sterile water at $1 \mathrm{mg} / \mathrm{mL}$ before storage at $-20{ }^{\circ} \mathrm{C}$. LEGEND MAX ${ }^{\mathrm{TM}}$ Enzyme-linked immunosorbent assay (ELISA) kits for rat TNF- $\alpha$ and IL-6 were from BioLegend (San Diego, CA, USA). ELISA kits for rat IL-10 were from Thermo Fisher Scientific (Waltham, MA, USA). P450-Glo CYP2B6 and CYP3A4 kits were from Promega (Madison, WI, USA). Human liver microsomes (HLMs) from a 200-donor pool of male and female donors were from Sekisui XenoTech (Kansas City, KS, USA).

\subsection{In Vitro P450 Inhibition}

Promega P450-Glo kits for CYP2B6 or CYP3A4 were used with pooled HLMs (200 subjects) to determine an $\mathrm{IC}_{50}$ for each extract, tea, or phytochemical. Test concentrations ranged from $0.01-600 \mu \mathrm{M}$ for each extract, tea, or phytochemical. A. annua extracts and teas were classified by their artemisinin content. For example, a $600 \mu \mathrm{M}$ DLA extract is an extract with artemisinin at a $600 \mu \mathrm{M}$ concentration. A. afra extracts and teas were made with a leaf dry weight equivalent to that used for $A$. annua extracts and teas such that a $600 \mu \mathrm{M} A$. afra extract was from the same leaf dry weight as a $600 \mu \mathrm{M}$ extract of $A$. annua. Assays followed manufacturer instructions and plates were read on a luminescence plate reader. Methanol, acetonitrile, or acetone were cosolvent vehicles at $\leq 1 \%(\mathrm{v} / \mathrm{v})$; a vehicle control was used for each comparison. $\mathrm{IC}_{50}$ values were determined through nonlinear regression using GraphPad Prism 7 (San Diego, CA, USA). All experiments had technical duplicates. Extracts, teas, and phytochemicals with an $\mathrm{IC}_{50}$ below $50 \mu \mathrm{M}$ were repeated in biological triplicate.

\subsection{Animals}

Sprague-Dawley rats (Charles River Laboratories, Wilmington, MA, USA) between 200-300 g (age 5-8 weeks for males and 6-9 weeks for females) were used for all animal studies and housed at the Worcester Polytechnic Institute Vivarium on a $14 \mathrm{~h}$ light, $10 \mathrm{~h}$ dark cycle with ad libitum food and water until the day before an experiment. Food was withheld $14 \mathrm{~h}$ before experiments as animal chow has been shown to alter artemisinin bioavailability [12]. All animal work was approved by the Worcester Polytechnic Institute Institutional Animal Care and Use Committee (IACUC Protocol 15-69 and 18-105) and in accordance with the NIH Guide for the Care and Use of Laboratory Animals. 


\subsection{In Vivo Artemisinin Tissue Distribution}

Artemisinin was delivered to rats po as either a slurry of powdered dried A. annua in water (DLA) or pure artemisinin in water with $12 \%$ DMSO. Artemisinin is essentially insoluble in water, so DMSO was used to enhance artemisinin solubility and achieve more consistent, replicable data. DLA-treated animals received $85 \mathrm{mg} / \mathrm{kg}$ dose of artemisinin in DLA slurry, the highest achievable dose that could be delivered while still conforming to animal welfare regulations. Initially we aimed to use the same dose of artemisinin in DLA and pure artemisinin-dosed animals, but preliminary experiments with a DLA-dosed rat and an artemisinin-dosed rat at $85 \mathrm{mg} / \mathrm{kg}$ showed no artemisinin was detectable in artemisinin treated animals. This was not surprising because pure artemisinin has very low oral bioavailability [29]; a previous study had to dose at $900 \mathrm{mg} / \mathrm{kg}$ of artemisinin to yield detectable artemisinin levels in the tissues [14]. Consequently, we increased the artemisinin dose in the control animals to $500 \mathrm{mg} / \mathrm{kg}$ and normalized amounts recovered in tissues, serum, urine, and feces to the delivered dose. After $1 \mathrm{~h}$, animals were euthanized by $\mathrm{CO}_{2}$ inhalation; tissues and blood were harvested immediately after death confirmation. For longer experiments, rats housed in metabolic cages for the duration of the $8 \mathrm{~h}$ time course had urine, feces, and $0.4 \mathrm{~mL}$ blood samples collected 1, 2, 4 and $8 \mathrm{~h}$ after gavage to track artemisinin and one of its liver metabolites, deoxyartemisinin. Tissues were collected and flash frozen on liquid nitrogen $8 \mathrm{~h}$ after gavage. Blood was collected in heparin-free serum collection tubes, allowed to clot $20 \mathrm{~min}$ before centrifugation at 1,500 $\mathrm{g}$ for $10 \mathrm{~min}$, and then stored at $-80{ }^{\circ} \mathrm{C}$ until extraction.

\subsection{Tissue Extraction}

Artemisinin and deoxyartemisinin were extracted from harvested tissues including heart, lungs, liver, spleen, kidneys, muscle, testes/ovaries, and brain. Briefly, a tissue sample was weighed, minced and then transferred to a Potter-Elvehjem tissue homogenizer. Water was added to yield a thick slurry and then homogenized. An aliquot was transferred into a new test tube and dichloromethane added at a 1:1 volume ratio. Samples were sonicated in a sonicating water bath for $30 \mathrm{~min}$ at ambient temperature and the organic layer decanted. The tissue residue was extracted twice again. Extracts were pooled, dried under nitrogen gas at ambient temperature, and stored at $-20{ }^{\circ} \mathrm{C}$ until analysis.

\subsection{Serum and Urine Extractions}

Serum and urine were thawed before extraction, aliquot transferred into a new tube, and combined with dichloromethane at a 2:1 ratio. Each sample was vortexed, placed in the sonicating water bath for $30 \mathrm{~min}$, the dichloromethane layer decanted, and extraction twice repeated. Extracts were pooled, dried under nitrogen, and stored at $-20{ }^{\circ} \mathrm{C}$ until analysis.

\subsection{Fecal Extractions}

Fecal samples collected at $1,2,4$ and $8 \mathrm{~h}$ were stored at $-80^{\circ} \mathrm{C}$ until extraction. Before extraction, samples were vacuum dried for $72 \mathrm{~h}$, ground with a mortar and pestle, wrapped in a tared Kimwipe, and weighed. Four milliliters dichloromethane was added to each sample in a test tube and then sonicated for $30 \mathrm{~min}$ and dried as described. Dried extracts were resuspended in $2 \mathrm{~mL}$ pentane and filtered through a $0.45 \mu \mathrm{m}$ PVDF syringe filter into a new test tube to remove particulates. After a second extraction, pooled extracts were filtered and stored at $-20{ }^{\circ} \mathrm{C}$ until analysis.

\subsection{Preparation of DLA Extracts and Teas}

Briefly, $1 \mathrm{~g}$ DLA (A. annua or A. afra) was twice extracted with $10 \mathrm{~mL}$ methanol, sonicated and filtered as previously described. The process was repeated with the same plant material and the extract was dried under a mild stream of nitrogen before artemisinin content was analyzed by GC-MS. Tea at $5 \mathrm{~g} / \mathrm{L}$ was prepared as described by Munyangi et al. (2018) [28]. 


\subsection{Artemisinin and Deoxyartemisinin Analyses}

Artemisinin and deoxyartemisinin were analyzed by gas chromatography mass spectrometry (GC-MS) as detailed previously [30]. Quantitation was based on standard curves from authenticated standards.

\subsection{Attenuation of Inflammation in Vivo}

A rat model of systemic inflammation was modified from Bison et al. 2009 [31] with four test groups: Injection control, LPS control, LPS+AN, and LPS+DLA. Injection controls received a single intraperitoneal injection of sterile water as vehicle control for LPS. LPS controls received an intraperitoneal injection of LPS in water at $2.5 \mathrm{mg} / \mathrm{kg}$ to induce a systemic inflammatory response. LPS+AN, and LPS+DLA animals received a $2.5 \mathrm{mg} / \mathrm{kg}$ intraperitoneal LPS dose, followed immediately by oral gavage of pure artemisinin or DLA at a $70 \mathrm{mg} / \mathrm{kg}$ artemisinin dose, respectively; $70 \mathrm{mg} / \mathrm{kg}$ was the maximum artemisinin dose deliverable as DLA. Pure artemisinin was delivered with $12 \%$ DMSO to enhance solubility. Blood samples $(0.4 \mathrm{~mL}$ ) were taken via tail snip at 0 (taken immediately before injection), 1, 2, 4 and $8 \mathrm{~h}$, clotted for $20 \mathrm{~min}$ at room temperature, and then centrifuged at $1500 \times g$ for $10 \mathrm{~min}$ to isolate serum. Serum was aliquoted and stored at $-80{ }^{\circ} \mathrm{C}$ before ELISA analysis performed according to manufacturer.

\subsection{Statistical Analysis}

Statistical analysis was performed using GraphPad Prism 7. IC $_{50}$ values were generated for CYP inhibition studies using nonlinear regression analysis in GraphPad Prism 7. Experiments were done in technical duplicate; three biological replicates were performed. $\mathrm{IC}_{50}$ values were compared to pure artemisinin by ANOVA. Each in vivo tissue distribution experiment compared the averages of three rats per group as permitted by the WPI IACUC. Students T-tests, used where appropriate, compared pure artemisinin to DLA dosed rats to determine statistical significance $(p<0.05)$. Each in vivo artemisinin elimination study compared the average of eight rats per group, as permitted by the WPI IACUC, and used students T-tests to compare DLA-dosed rats to rats dosed with pure artemisinin. Each in vivo inflammation experiment compared the averages of 5-6 rats per group except for the injection control in which there were three rats as was permitted by the WPI IACUC. ANOVA was used where appropriate to compare pure artemisinin- and DLA-dosed rats to LPS controls in inflammation studies.

\section{Results}

\subsection{Inhibition of CYP2B6 and CYP3A4 by DLA}

Extracts (DLA me and A. aframe) and teas (DLA tea and A. afratea) of both A. annua and A. afra inhibited both CYP2B6 and CYP3A4 activity (Table 1). All extracts and teas had a lower $\mathrm{IC}_{50}$ than that for artemisinin alone, indicating that compounds other than artemisinin from DLA inhibited CYP2B6 and CYP3A4 activity. In addition to extracts and teas, some individually tested phytochemicals had inhibitory activity, but few were as strong as the whole extract (Table 1).

\subsection{Artemisinin Delivered as DLA Increased Bioavailability in Some Tissues}

To determine how DLA alters distribution of artemisinin to tissues, we orally gavaged rats with DLA or pure artemisinin and measured artemisinin content. Compared to artemisinin-dosed males artemisinin in DLA-dosed rats at $1 \mathrm{~h}$ had significantly more artemisinin in the heart, muscle and serum (Figure 2A), while DLA-dosed females had significantly more artemisinin in the heart, lungs, liver, muscle, brain tissue, and serum (Figure 2B). In males, artemisinin accumulated mostly in serum followed by lungs, heart, liver, brain, spleen, muscle, and kidneys (Figure 2A). DLA-delivered 
artemisinin has greater bioavailability $[7,8,12]$, so we expected more artemisinin in DLA-treated tissues; this was confirmed, as shown in Figure 2.

Table 1. Inhibition of CYP2B6 and CYP3A4 by DLA extracts, teas and phytochemicals.

\begin{tabular}{ccc}
\hline Phytochemical or Extract & CYP2B6 IC $_{\mathbf{5 0}}(\mu \mathrm{M})$ & CYP3A4 IC $_{50}(\mu \mathrm{M})$ \\
\hline DLA $_{\text {me }}$ & $6.07^{* *}$ & $4.93^{\#}$ \\
DLA tea & $2.31^{* *}$ & $5.67^{\#}$ \\
A. aframe & $7.89^{* *}$ & $5.18^{\#}$ \\
A. afratea & $11.27^{* *}$ & $5.59^{\#}$ \\
Arteannuin B & $9.36^{* *}$ & $12.33^{\#}$ \\
Quercetin & $19.86^{*}$ & $4.59^{\#}$ \\
Artemisinin & 27.75 & $>600$ \\
Chrysosplenol-D & 52.06 & $22.96^{\#}$ \\
Chrysosplenetin & 74.67 & $21.68^{\#}$ \\
Kaempferol & $>600$ & $33.84^{\#}$ \\
Deoxyartemisinin & 110.86 & $>600$ \\
Camphor (+) & 119.01 & $>600$ \\
Artemisinic Acid & 122.83 & $239.5^{\#}$ \\
Camphor (-) & 141.93 & $>600$ \\
Scopoletin & $>600$ & $410.19^{\#}$ \\
Chlorogenic Acid & $>600$ & $>600$ \\
\hline
\end{tabular}

* $\mathrm{IC}_{50}$ significantly lower than artemisinin; $p \leq 0.05 ;{ }^{* *} p \leq 0.0001 ; n=3$. ${ }^{\#} \mathrm{IC}_{50}$ lower than artemisinin but no statistical difference determined because the artemisinin $\mathrm{IC}_{50}$ is higher than the highest tested concentration $(600 \mu \mathrm{M})$.

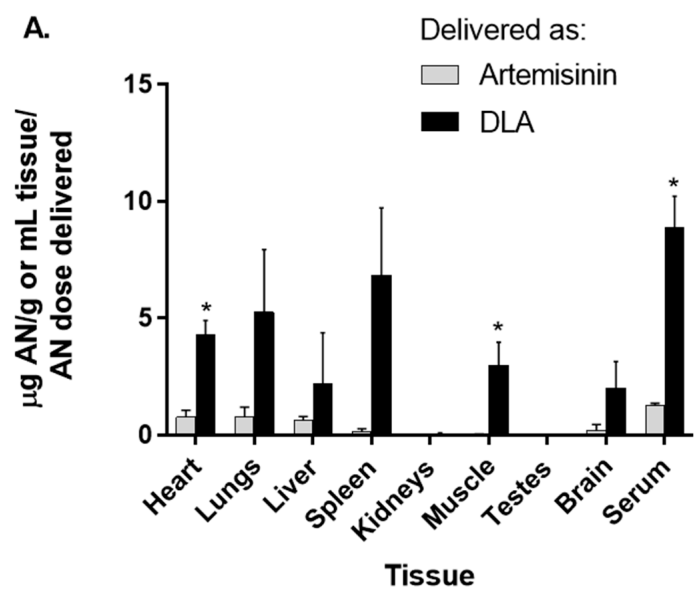

B.

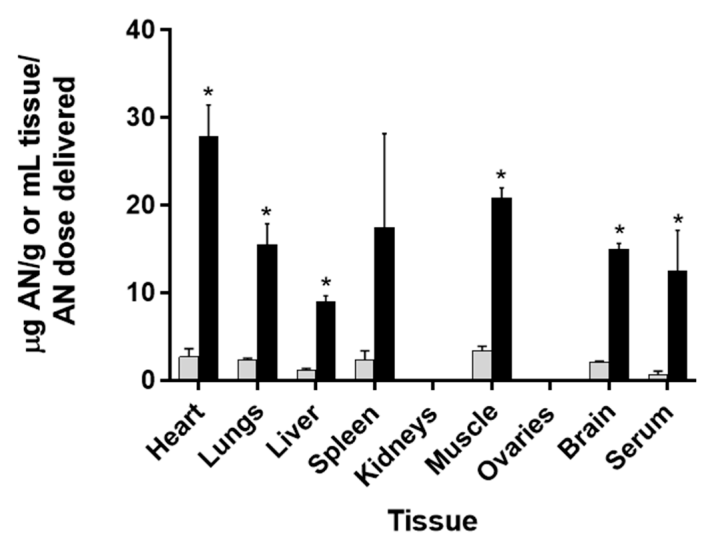

Figure 2. Artemisinin distribution in tissues and serum of male $(\mathbf{A})$ and female $(\mathbf{B})$ rats after oral delivery of artemisinin as dried leaf $A$. апnиа (DLA) or pure artemisinin. $n=3,{ }^{*} ; p \leq 0.05$; error bars $=$ SEM. 
While artemisinin distributed well to most tissues, it cleared by $8 \mathrm{~h}$. Neither artemisinin nor deoxyartemisinin was detectable in any tissues or serum $8 \mathrm{~h}$ postgavage. Serum was also collected at 2 and $4 \mathrm{~h}$ postgavage; however, neither artemisinin nor deoxyartemisinin was detectable in either gender. In male rats, heart, liver, and muscle had a significant difference in deoxyartemisinin distribution between DLA and pure artemisinin-treatments (Figure 3A). In female rats, however, there was a significant difference in deoxyartemisinin distribution to heart, lungs, spleen, muscle, brain, and serum (Figure 3B). DLA contains a small amount of deoxyartemisinin (7.5 mg/kg dose), so deoxyartemisinin detected in animals treated with DLA is likely not all from liver metabolism. Animals given pure artemisinin, however, did not receive any deoxyartemisinin, so any deoxyartemisinin detected in their tissues was due solely to artemisinin liver metabolism.

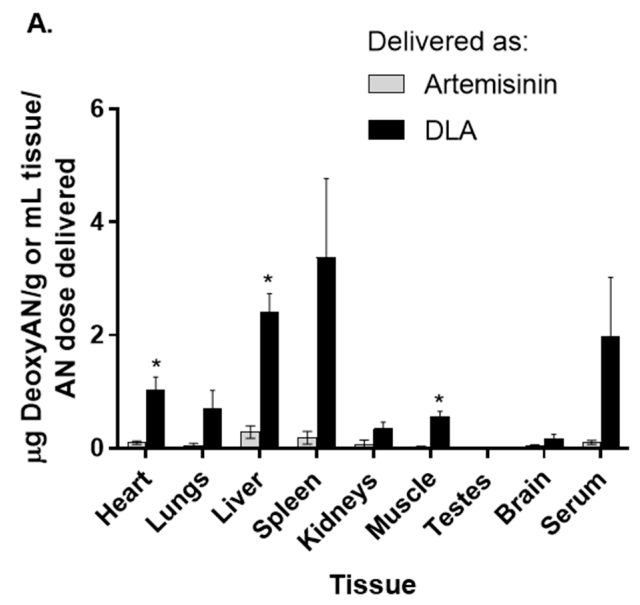

B.

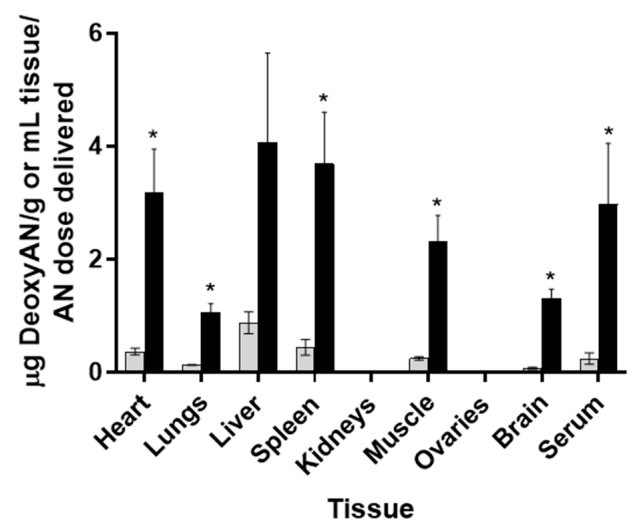

Figure 3. Deoxyartemisinin (deoxyAN) distribution in tissues and serum of male (A) and female (B) rats after oral delivery of artemisinin as dried leaf $A$. annua (DLA) or pure artemisinin. $n=3,{ }^{*} ; p \leq 0.05$; error bars $=$ SEM.

Distribution of artemisinin was significantly different in several tissues between males and females regardless of delivery mode (Table 2 ). At $1 \mathrm{~h}$, females gavaged with pure artemisinin had more artemisinin in lungs, liver, muscle, and brain tissue than males (Table 2). Similarly, females gavaged with DLA had more artemisinin in the heart, lungs, liver, muscle, and brain tissue at $1 \mathrm{~h}$ compared to males (Table 2). In almost all tissues, although not always statistically significant, artemisinin and deoxyartemisinin were more abundant in females than males at $1 \mathrm{~h}$. These data suggest females absorb and distribute artemisinin throughout the body more efficiently than males. There was, however, no difference in serum artemisinin levels at $1 \mathrm{~h}$ between female and male rats given either pure artemisinin or DLA (Table 2), suggesting that tissue distribution of artemisinin-but not absorption into the blood-is gender dependent. 
Table 2. Gender-specific differences in artemisinin and deoxyartemisinin distribution to tissues in artemisinin- and DLA-treated rats.

\begin{tabular}{|c|c|c|c|c|c|c|c|c|}
\hline \multirow[b]{3}{*}{ Tissue } & \multicolumn{4}{|c|}{ Artemisinin Treated } & \multicolumn{4}{|c|}{ DLA Treated } \\
\hline & \multicolumn{2}{|c|}{$\begin{array}{c}\text { Artemisinin ( } \mu \text { g AN/g tissue/g AN } \\
\text { delivered } \pm \text { SD) }\end{array}$} & \multicolumn{2}{|c|}{$\begin{array}{c}\text { Deoxyartemisinin ( } \mu \text { g DeoxyAN/g } \\
\text { tissue/g AN delivered } \pm \text { SD) }\end{array}$} & \multicolumn{2}{|c|}{$\begin{array}{c}\text { Artemisinin ( } \mu \text { g AN/g tissue/g AN } \\
\text { delivered } \pm \text { SD) }\end{array}$} & \multicolumn{2}{|c|}{$\begin{array}{l}\text { Deoxyartemisinin ( } \mu \text { g DeoxyAN/g } \\
\text { tissue/g AN delivered } \pm \text { SD) }\end{array}$} \\
\hline & Male & Female & Male & Female & Male & Female & Male & Female \\
\hline Heart & $0.77 \pm 0.49$ & $2.70 \pm 1.87$ & $0.097 \pm 0.050$ & $0.37 \pm 0.12$ * & $4.30 \pm 1.06$ & $27.81 \pm 7.25$ * & $1.02 \pm 0.42$ & $3.18 \pm 1.54$ \\
\hline Lungs & $0.79 \pm 0.69$ & $2.37 \pm 0.26^{*}$ & $0.055 \pm 0.054$ & $0.13 \pm 0.018 *$ & $5.26 \pm 4.64$ & $15.56 \pm 4.63 *$ & $0.70 \pm 0.65$ & $1.06 \pm 0.31$ \\
\hline Liver & $0.62 \pm 0.30$ & $1.23 \pm 0.26^{*}$ & $0.29 \pm 0.19$ & $0.88 \pm 0.39$ & $2.19 \pm 3.79$ & $8.96 \pm 1.40 *$ & $2.42 \pm 0.55$ & $4.06 \pm 3.18$ \\
\hline Spleen & $0.14 \pm 0.24$ & $2.37 \pm 2.05$ & $0.19 \pm 0.20$ & $0.44 \pm 0.28$ & $6.82 \pm 5.03$ & $17.49 \pm 21.37$ & $3.38 \pm 2.79$ & $3.69 \pm 1.83$ \\
\hline Kidneys & $0.00 \pm 0.00$ & $0.00 \pm 0.00$ & $0.074 \pm 0.12$ & $0.00 \pm 0.00$ & $0.048 \pm 0.096$ & $0.00 \pm 0.00$ & $0.35 \pm 0.23$ & $0.00 \pm 0.00^{*}$ \\
\hline Muscle & $0.019 \pm 0.034$ & $3.41 \pm 0.85^{*}$ & $0.025 \pm 0.018$ & $0.25 \pm 0.049 *$ & $2.99 \pm 1.68$ & $20.84 \pm 2.26 *$ & $0.57 \pm 0.15$ & $2.31 \pm 0.93 *$ \\
\hline Testes/Ovaries & $0.00 \pm 0.00$ & $0.00 \pm 0.00$ & $0.00 \pm 0.00$ & $0.00 \pm 0.00$ & $0.00 \pm 0.00$ & $0.00 \pm 0.00$ & $0.00 \pm 0.00$ & $0.00 \pm 0.00$ \\
\hline Brain & $0.22 \pm 0.38$ & $2.05 \pm 0.35 *$ & $0.038 \pm 0.043$ & $0.075 \pm 0.034$ & $2.02 \pm 1.96$ & $15.00 \pm 1.31 *$ & $0.18 \pm 0.12$ & $1.30 \pm 0.36^{*}$ \\
\hline Serum & $1.29 \pm 0.11$ & $0.67 \pm 0.75$ & $0.10 \pm 0.061$ & $0.24 \pm 0.20$ & $8.90 \pm 2.63$ & $12.49 \pm 9.34$ & $1.97 \pm 2.10$ & $2.97 \pm 2.17$ \\
\hline
\end{tabular}

$\mathrm{AN}$, artemisinin; deoxyAN, deoxyartemisinin; $\mathrm{SD}$, standard deviation; $n=3 ;{ }^{*}, p \leq 0.05$ when compared to opposite gender. 


\subsection{Artemisinin Elimination through the Urine is Gender-Dependent}

To compare artemisinin elimination from DLA vs. pure artemisinin, urine collected over $8 \mathrm{~h}$ from female rats showed significantly more artemisinin elimination than males (Figure 4C). Because there is some deoxyartemisinin naturally present in DLA, those animals received $7.5 \mathrm{mg} / \mathrm{kg}$ of deoxyartemisinin in the DLA gavage. Animals treated with pure artemisinin did not receive any deoxyartemisinin. When comparing genders, females had more artemisinin in urine than males when given DLA. Females also had more deoxyartemisinin in urine than males, regardless of how artemisinin was delivered (Figure 5).

A.

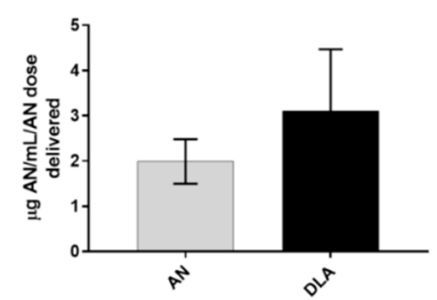

c.

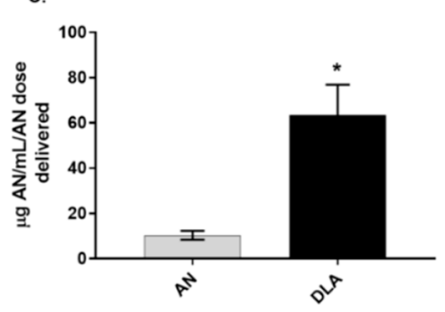

B.

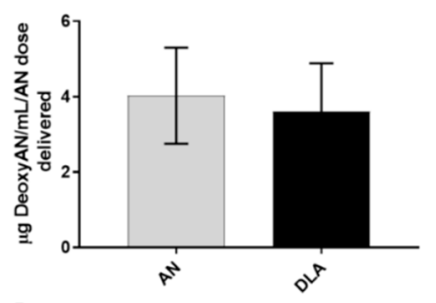

D.

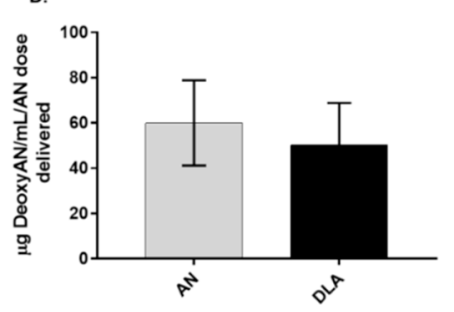

Figure 4. Artemisinin (AN) and deoxyartemisinin (DeoxyAN) accumulation in urine of male (A,B) and female (C,D) rats after oral delivery of artemisinin as dried leaf $A$. annua (DLA) or pure artemisinin. $n$ $=8,{ }^{*} ; p \leq 0.05$; error bars $=$ SEM.

A.

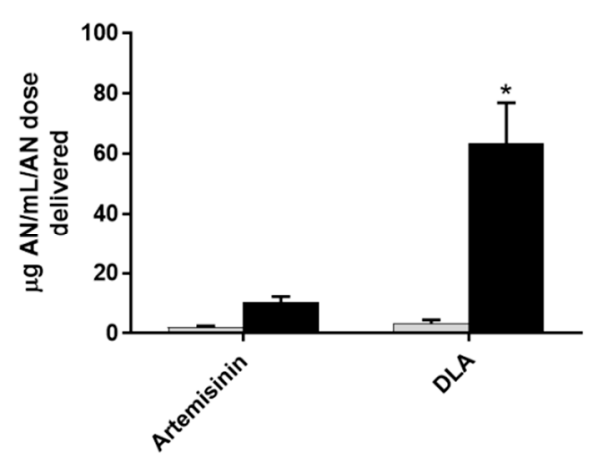

AN Delivery Method
B.
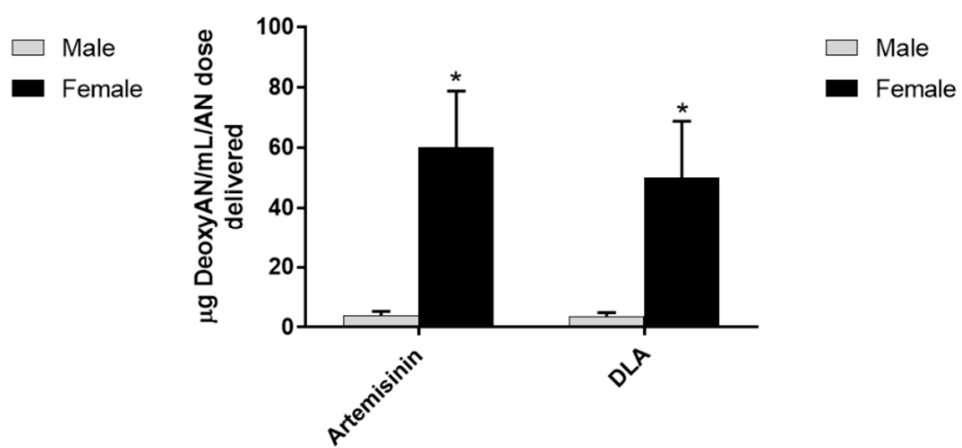

AN Delivery Method

Figure 5. Artemisinin (A) and deoxyartemisinin (B) found in the urine of male and female rats orally gavaged with either pure artemisinin or dried leaves of $A$. annua (DLA). $n=8$; ${ }^{*} p \leq 0.05$; error bars $=$ SEM

\subsection{Artemisinin Elimination through Feces}

Artemisinin and deoxyartemisinin was measured in the feces of rats up to $8 \mathrm{~h}$ after oral gavage with DLA or pure artemisinin (Supplementary Figure S1). As expected, most artemisinin and deoxyartemisinin was eliminated through feces, and was detected at later time points. Although total artemisinin and deoxyartemisinin was summed from the feces over $8 \mathrm{~h}$, there were no significant differences in either artemisinin or deoxyartemisinin between DLA and pure artemisinin dosed animals 
(Supplementary Figure S2). We also measured fecal microbiome samples and found neither artemisinin nor DLA treatment affected the microbial population (Supplementary Table S1).

\subsection{Attenuation of Inflammation by Artemisinin and DLA}

As expected, both male and female LPS controls responded to LPS challenge with a systemic inflammatory response. Serum TNF- $\alpha$ levels spiked quickly and peaked at about 4,600 pg/mL for males (Figure 6A) and 5,300 pg/mL for females (Figure 6B) at $1 \mathrm{hr}$ after LPS injection. The spike in TNF- $\alpha$ was followed by a spike in serum IL-6 at 2 hours after LPS injection with males peaking at about 15,400 pg/mL (Figure 6C) and females peaking at about 25,100 pg/mL (Figure 6D). Interestingly, only DLA was effective at reducing serum TNF- $\alpha$ levels in males and only at the $1 \mathrm{~h}$ time point, while both DLA and pure artemisinin significantly reduced serum TNF- $\alpha$ levels at 1 and $2 \mathrm{~h}$ postinjection in females (Figure 6). Although artemisinin and DLA were effective at reducing TNF- $\alpha$ production in females, neither treatment had any effect on IL-6 serum levels in females. In males, however, DLA reduced serum IL-6 levels at 2 and $4 \mathrm{~h}$ postinjection. Overall, DLA was more effective at reducing inflammatory cytokine production in both genders, as expected based on the diversity of anti-inflammatory phytochemicals in DLA.
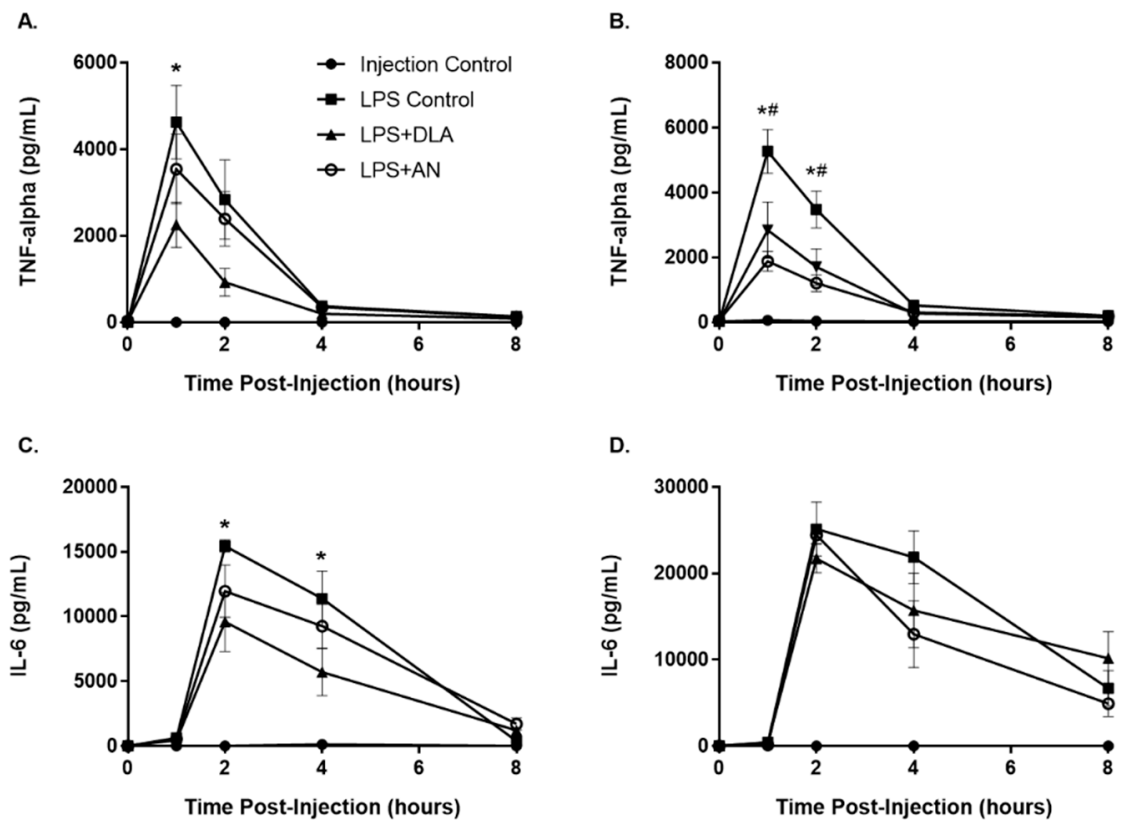

Figure 6. Production of proinflammatory cytokines TNF- $\alpha$ and IL-6 in male (A,C) and female (B,D) rats after LPS challenge and treatment with either pure artemisinin (AN) or DLA (equal artemisinin doses). $n=5-6$ for experimental conditions, $n=3$ for injection control; error bars = SEM; ${ }^{*}, p \leq 0.05$ when comparing LPS+DLA to LPS Control; \#, $p \leq 0.05$ when comparing LPS+AN to LPS Control.

We also measured the effects of DLA and pure artemisinin treatment on the anti-inflammatory cytokine IL-10. However, there were no significant differences in serum IL-10 levels, regardless of treatment (Figure S2).

\section{Discussion}

A. annua produces many phytochemicals including kaempferol, chrysosplenetin, and quercetin [26,32] that have already been shown to inhibit either cytochrome P450 isoform 3A4 or 2B6, which are responsible for artemisinin metabolism in humans [10,33-36]. We hypothesized that the increased bioavailability of artemisinin afforded by DLA-delivery [9] is partly due to inhibition of hepatic metabolism by phytochemicals produced by DLA. The data presented in Section 3.1 confirm that phytochemicals present in DLA do inhibit liver P450s and this inhibition is part of the mechanism 
that leads to the previously reported increased bioavailability of DLA-delivered artemisinin [9]. Furthermore, since no individually tested phytochemicals produced stronger inhibition than the DLA extract, this inhibition is not solely due to any of the measured phytochemicals. It could, however, be from other as yet unidentified phytochemicals or a complex mixture of phytochemicals with moderate inhibitory activity. Future experiments will involve bioassay-guided fractionation of DLA extract to determine if there are any singular phytochemicals with potent inhibitory P450 activity.

In mice, artemisinin reaches peak serum concentration at $1 \mathrm{~h}$ postgavage [12]. Using TLC, Xinyi et al. (1985) showed orally delivered artemisinin accumulated mostly in the liver followed by

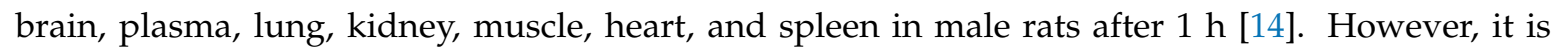
unclear whether their TLC method could resolve artemisinin from deoxyartemisinin, an important liver metabolite. Here, using GC-MS analysis, we expanded on those results by determining differences when artemisinin was instead delivered as DLA and by comparing gender differences. DLA-delivered artemisinin distributed in higher quantities to many tissues in males and females. This was not surprising, given that DLA-delivered artemisinin was known to have increased bioavailability $[9,12,13]$. Interestingly, female rats had both better absorption of artemisinin into several tissues (Table 2) and higher amounts of artemisinin in their urine when given DLA (Figure 5). These data are consistent with a previous report showing females to have significantly decreased artemisinin metabolism compared to males in vitro [15].

The enhanced tissue distribution seen here and increased bioavailability afforded by DLA shown in the literature $[9,12,13]$ led us to hypothesize DLA would be a more potent anti-inflammatory therapeutic than pure artemisinin. Indeed, the results shown in Figure 6 confirm this hypothesis. The enhanced efficacy against inflammatory cytokine production was likely due to increased bioavailability of artemisinin afforded by the inhibition of CYP2B6 and CYP3A4 by DLA phytochemicals. However, it is also plausible some DLA phytochemicals have their own inherent anti-inflammatory activity, and thus act additively with artemisinin to suppress inflammatory cytokine production. It is also possible that both mechanisms are in play. In either case, the benefit of the whole plant-based treatment is evident. While gender differences were expected, it was surprising that IL-6 production was affected neither by DLA nor pure artemisinin treatment in females, especially considering artemisinin is better absorbed and less efficiently metabolized in females compared to males [15].

We also posited that if DLA reduced inflammation, the reduction could occur by increasing anti-inflammatory cytokine production, e.g., IL-10. However, the results shown in Supplementary Figure S2 suggest that is unlikely. More likely is that DLA phytochemicals interact with molecular pathways responsible for inflammatory cytokine production, e.g., NF- $\mathrm{BB}$ and MAPK signaling, and should be further investigated in vitro with DLA extracts and individual phytochemicals.

Taken together, these results should be considered for potential drug-drug interactions with other medications taken by patients consuming A. annua. Consistent with the human pharmacokinetic study of Rath et al. (2004) [13], our results also indicate that delivery of artemisinin via per os consumption of $A$. annua can exceed the minimum antimalarial threshold of 9-10 $\mu \mathrm{g} / \mathrm{L}$ blood [37] and should therefore obviate some concerns expressed by WHO $(2012,2019)[38,39]$ regarding inadequate delivery of artemisinin from per os consumption of $A$. annua.

\section{Conclusions}

Although DLA extracts, teas, and phytochemicals inhibited CYP2B6 and CYP3A4, thereby showing that phytochemicals in DLA inhibit CYP450 enzymes that metabolize artemisinin, almost nothing is known about its tissue distribution and elimination. While the ADME of pure artemisinin was briefly explored previously by TLC [14], it was not compared to artemisinin delivered as DLA. Here, we showed that distribution of artemisinin to several tissues and serum significantly increased when delivered as DLA in male and female rats. Furthermore, the data suggest that artemisinin is differentially eliminated in males compared to females and differentially metabolized from DLA vs. pure artemisinin. More artemisinin therefore passes into the serum. Although we showed DLA is 
an effective anti-inflammatory agent in vivo, reducing TNF- $\alpha$ and IL- 6 in males, in females, results were not as definitive because neither DLA nor pure artemisinin reduced IL-6 production, but did reduce TNF- $\alpha$. Finally, neither artemisinin nor DLA affected IL-10 production, indicating that DLA and artemisinin likely reduce inflammation by inhibiting molecular signaling pathways responsible for proinflammatory cytokine production. Inhibition of the NF- $\mathrm{KB}$ and MAPK signaling cascade pathways by DLA extracts and phytochemicals require further investigation. Overall, these results enhance our understanding of how artemisinin delivered from $A$. annua is more bioavailable than when delivered as a pure drug.

Supplementary Materials: The following are available online at http://www.mdpi.com/2218-273X/10/2/254/s1, Figure S1: Artemisinin (AN) and deoxyartemisinin (DeoxyAN) accumulation in feces of male (A,B) and female (C,D) rats after oral delivery of artemisinin as dried leaf A. annua (DLA) or pure artemisinin. $n=7-8,{ }^{*} ; p<0.05$; error bars = SEM., Figure S2: Production of anti-inflammatory cytokine IL-10 in male (A) and female (B) rats after LPS challenge and treatment with either pure artemisinin (AN) or DLA (equal artemisinin doses). $n=5-6$ for experimental conditions, $n=3$ for injection control; error bars = SEM, Table S1: Microbiome population by Phylum $1 \mathrm{~h}$ and $8 \mathrm{~h}$ after treatment with DLA or pure artemisinin in male rats.

Author Contributions: Conceptualization, M.R.D. and P.J.W.; methodology, M.R.D.; formal analysis, M.R.D.; investigation, M.R.D. and A.M.; writing—original draft preparation, M.R.D.; writing—review and editing, M.R.D., A.M., and P.J.W.; supervision, P.J.W.; project administration, P.J.W.; funding acquisition, P.J.W. All authors have read and agreed to the published version of the manuscript.

Funding: This research was funded by The National Center for Complementary and Integrative Health, award number NIH-2R15AT008277-02. The content is solely the responsibility of the authors and does not necessarily represent the official views of the National Center for Complementary and Integrative Health or the National Institutes of Health.

Acknowledgments: The authors thank Miguel Norden and Virender Chandna of Worcester Polytechnic Institute for training Desrosiers in all procedures with rats as well as assistance with intraperitoneal injections. We also thank vivarium staff for help with animal welfare and upkeep. We thank David Greenblatt and Mia Angeli of Tufts University School of Medicine for training in liver microsome studies. We thank M. Andrea Azcarate-Peril, Jakub Kwintkiewicz, and Kshipra Chandrashekhar of the University of North Carolina Chapel Hill Microbiome Core Facility for help with 16s rRNA sequencing. We also thank Melissa Towler of Worcester Polytechnic Institute for phytochemical analysis of DLA.

Conflicts of Interest: The authors declare no conflict of interest. The funders had no role in the design of the study; in the collection, analyses, or interpretation of data; in the writing of the manuscript, or in the decision to publish the results.

\section{References}

1. WHO. Malaria. Available online: http://www.who.int/mediacentre/factsheets/fs094/en/ (accessed on 23 December 2019).

2. WHO. Guidelines for the treatment of malaria. Available online: http://apps.who.int/iris/bitstream/10665/ 162441/1/9789241549127_eng.pdf?ua=1\&ua=1 (accessed on 23 December 2019).

3. Olsson, M.E.; Olofsson, L.M.; Lindahl, A.L.; Lundgren, A.; Brodelius, M.; Brodelius, P.E. Localization of enzymes of artemisinin biosynthesis to the apical cells of glandular secretory trichomes of Artemisia annua L. Phytochemistry 2009, 70, 1123-1128. [CrossRef]

4. Hsu, E. The history of qing hao in the Chinese materia medica. Trans. R. Soc. Trop. Med. Hyg. 2006, 100, 505-508. [CrossRef] [PubMed]

5. Munyangi, J.; Cornet-Vernet, L.; Idumbo, M.; Lu, C.; Lutgen, P.; Perronne, C.; Ngombe, N.; Bianga, J.; Mupenda, B.; Lalukala, P.; et al. Artemisia annua and Artemisia afra tea infusions vs. artesunate-amodiaquine (ASAQ) in treating Plasmodium falciparum malaria in a large scale, double blind, randomized clinical trial. Phytomedicine 2019, 57, 49-56. [CrossRef] [PubMed]

6. ICIPE. Whole-leaf Artemisia Annua-based Antimalarial Drug: Report on Proof-of-Concepts Studies; ICIPE: Nairobi, Kenya, 2005.

7. Desrosiers, M.R.; Weathers, P.J. Effect of leaf digestion and artemisinin solubility for use in oral consumption of dried Artemisia annua leaves to treat malaria. J. Ethnopharmacol. 2016, 190, 313-318. [CrossRef] [PubMed]

8. Desrosiers, M.R.; Weathers, P.J. Artemisinin permeability via Caco-2 cells increases after simulated digestion of Artemisia annua leaves. J. Ethnopharmacol. 2018, 210, 254-259. [CrossRef] [PubMed] 
9. Weathers, P.J.; Arsenault, P.R.; Covello, P.S.; McMickle, A.; Teoh, K.H.; Reed, D.W. Artemisinin production in Artemisia annua: Studies in planta and results of a novel delivery method for treating malaria and other neglected diseases. Phytochem. Rev. 2011, 10, 173-183. [CrossRef] [PubMed]

10. Svensson, U.S.H.; Ashton, M. Identification of the human cytochrome P450 enzymes involved in the in vitro metabolism of artemisinin. Br. J. Clin. Pharmacol. 1999, 48, 528-535. [CrossRef]

11. Lee, I.S.; Hufford, C.D. Metabolism of antimalarial sesquiterpene lactones. Pharmacol. Ther. 1990, 48, 345-355. [CrossRef]

12. Weathers, P.J.; Elfawal, M.A.; Towler, M.J.; Acquaah-Mensah, G.K.; Rich, S.M. Pharmacokinetics of artemisinin delivered by oral consumption of Artemisia annua dried leaves in healthy vs. Plasmodium chabaudi-infected mice. J. Ethnopharmacol. 2014, 153, 732-736. [CrossRef]

13. Räth, K.; Taxis, K.; Walz, G.; Gleiter, C.H.; Li, S.M.; Heide, L. Pharmacokinetic study of artemisinin after oral intake of a traditional preparation of Artemisia annua L.(annual wormwood). Am. J. Trop. Med. Hyg. 2004, 70, 128-132. [CrossRef]

14. Xinyi, N.; Liyi, H.O.; Zhihong, R.; Zhenyu, S. Metabolic fate of Qinghaosu in rats; a new TLC densitometric method for its determination in biological material. Eur. J. Drug Metab. Pharmacokinet. 1985, 10, 55-59. [CrossRef] [PubMed]

15. Ashton, M. Quantitative in vivo and in vitro sex differences in artemisinin metabolism in rat. Xenobiotica 1999, 29, 195-204. [CrossRef] [PubMed]

16. Wang, K.S.; Li, J.; Wang, Z.; Mi, C.; Ma, J.; Piao, L.X.; Xu, G.H.; Li, X.; Jin, X. Artemisinin inhibits inflammatory response via regulating NF-kB and MAPK signaling pathways. Immunopharmacol. Immunotoxicol. 2017, 39, 28-36. [CrossRef] [PubMed]

17. Kim, W.S.; Choi, W.J.; Lee, S.; Kim, W.J.; Lee, D.C.; Sohn, U.D.; Shin, H.S.; Kim, W. Anti-inflammatory, Antioxidant and Antimicrobial Effects of Artemisinin Extracts from Artemisia annua L. Kor. J. Physiol. Pharmacol. 2015, 19, 21-27. [CrossRef]

18. Rathee, P.; Chaudhary, H.; Rathee, S.; Rathee, D.; Kumar, V.; Kohli, K. Mechanism of action of flavonoids as anti-inflammatory agents: a review. Inflamm. Allergy Drug Targets 2009, 8, 229-235. [CrossRef]

19. De Cássia da Silveira e Sá, R.; Andrade, L.N.; de Sousa, D.P. A review on anti-inflammatory activity of monoterpenes. Molecules 2013, 18, 1227-1254. [CrossRef]

20. Li, Y.J.; Guo, Y.; Yang, Q.; Weng, X.G.; Yang, L.; Wang, Y.J.; Chen, Y.; Zhang, D.; Li, Q.; Liu, X.C.; et al. Flavonoids casticin and chrysosplenol D from Artemisia annua L. inhibit inflammation in vitro and in vivo. Toxicol. Appl. Pharmacol. 2015, 286, 151-158. [CrossRef]

21. Juergens, U.R.; Engelen, T.; Racké, K.; Stöber, M.; Gillissen, A.; Vetter, H. Inhibitory activity of 1, 8-cineol (eucalyptol) on cytokine production in cultured human lymphocytes and monocytes. Pulm. Pharmacol. Ther. 2004, 17, 281-287. [CrossRef]

22. Melillo de Magalhães, P.; Dupont, I.; Hendrickx, A.; Joly, A.; Raas, T.; Dessy, S.; Sergent, T.; Schneider, Y.-J. Anti-inflammatory effect and modulation of cytochrome P450 activities by Artemisia annua tea infusions in human intestinal Caco-2 cells. Food Chem. 2012, 134, 864-871. [CrossRef]

23. Weathers, P.J.; Jordan, N.J.; Lasin, P.; Towler, M.J. Simulated digestion of dried leaves of Artemisia annua consumed as a treatment (pACT) for malaria. J. Ethnopharmacol. 2014, 151, 858-863. [CrossRef]

24. Weathers, P.J.; Towler, M.J. The flavonoids casticin and artemetin are poorly extracted and are unstable in an Artemisia annua tea infusion. Planta Med. 2012, 78, 1024-1026. [CrossRef] [PubMed]

25. Arvouet-Grand, A.; Vennat, B.; Pourrat, A.; Legret, P. Standardization of propolis extract and identification of principal constituents. J. Pharm. Belg. 1994, 49, 462-468. [PubMed]

26. Weathers, P.J.; Towler, M.J. Changes in key constituents of clonally propagated Artemisia annua L. during preparation of compressed leaf tablets for possible therapeutic use. Ind. Crop. Prod. 2014, 62, 173-178. [CrossRef] [PubMed]

27. Gruessner, B.M.; Cornet-Vernet, L.; Desrosiers, M.R.; Lutgen, P.; Towler, M.J.; Weathers, P.J. It is not just artemisinin: Artemisia sp. for treating diseases including malaria and schistosomiasis. Phytochem. Rev. 2019. [CrossRef]

28. Munyangi, J.; Cornet-Vernet, L.; Idumbo, M.; Lu, C.; Lutgen, P.; Perronne, C.; Ngombe, N.; Bianga, J.; Mupenda, B.; Lalukala, P.; et al. Effect of Artemisia annua and Artemisia afra tea infusions on schistosomiasis in a large clinical trial. Phytomedicine 2018, 51, 233-240. [CrossRef] 
29. Titulaer, H.A.C.; Zuidema, J.; Kager, P.A.; Wetsteyn, J.; Lugt, C.B.; Merkus, F. The pharmacokinetics of artemisinin after oral, intramuscular and rectal administration to volunteers. J. Pharm. Pharmacol. 1990, 42, 810-813. [CrossRef]

30. Towler, M.J.; Weathers, P.J. Variations in key artemisinic and other metabolites throughout plant development in Artemisia annua L. for potential therapeutic use. Ind. Crop. Prod. 2015, 67, 185-191. [CrossRef]

31. Bison, S.; Carboni, L.; Arban, R.; Bate, S.; Gerrard, P.A.; Razzoli, M. Differential behavioral, physiological, and hormonal sensitivity to LPS challenge in rats. Int. J. Interferon Cytokine Mediat. Res. 2009, 1, 1-13. [CrossRef]

32. Ferreira, J.F.S.; Luthria, D.L.; Sasaki, T.; Heyerick, A. Flavonoids from Artemisia annua L. as antioxidants and their potential synergism with artemisinin against malaria and cancer. Molecules 2010, 15, 3135-3170. [CrossRef]

33. Lau, A.J.; Chang, T.K.H. Inhibition of Human CYP2B6-Catalyzed Bupropion Hydroxylation by Ginkgo biloba Extract: Effect of Terpene Trilactones and Flavonols. Drug Metab. Dispos. 2009, 37, 1931. [CrossRef]

34. Wei, S.; Ji, H.; Yang, B.; Ma, L.; Bei, Z.; Li, X.; Dang, H.; Yang, X.; Liu, C.; Wu, X.; et al. Impact of chrysosplenetin on the pharmacokinetics and anti-malarial efficacy of artemisinin against Plasmodium berghei as well as in vitro CYP450 enzymatic activities in rat liver microsome. Malar. J. 2015, 14, 432. [CrossRef] [PubMed]

35. Sergent, T.; Dupont, I.; Van Der Heiden, E.; Scippo, M.L.; Pussemier, L.; Larondelle, Y.; Schneider, Y.J. CYP1A1 and CYP3A4 modulation by dietary flavonoids in human intestinal Caco-2 cells. Toxicol. Lett. 2009, 191, 216-222. [CrossRef] [PubMed]

36. Choi, J.S.; Piao, Y.J.; Kang, K.W. Effects of quercetin on the bioavailability of doxorubicin in rats: Role of CYP3A4 and P-gp inhibition by quercetin. Arch. Pharm. Res. 2011, 34, 607-613. [CrossRef] [PubMed]

37. Alin, M.H.; Bjorkman, A. Concentration and time dependency of artemisinin efficacy against Plasmodium falciparum in vitro. Am. J. Trop. Med. Hyg. 1994, 50, 771-776. [CrossRef] [PubMed]

38. WHO. Position Statement. Effectiveness of Non-Pharmaceutical Forms of Artemisia annua L. against malaria. Available online: https:/www.who.int/malaria/diagnosis_treatment/position_statement_herbal_remedy_ artemisia_annua_1.pdf?ua=1 (accessed on 23 December 2019).

39. WHO. The Use of Non-pharmaceutical Forms of Artemisia. Available online: https://www.who.int/ publications-detail/the-use-of-non-pharmaceutical-forms-of-artemisia (accessed on 23 December 2019).

(C) 2020 by the authors. Licensee MDPI, Basel, Switzerland. This article is an open access article distributed under the terms and conditions of the Creative Commons Attribution (CC BY) license (http://creativecommons.org/licenses/by/4.0/). 